\title{
Management of hypoadrenocorticism (Addison's disease) in dogs
}

This article was published in the following Dove Press journal: Veterinary Medicine: Research and Reports

\author{
Patty Lathan' \\ Ann L Thompson ${ }^{2}$ \\ 'College of Veterinary Medicine, \\ Mississippi State University, \\ Starkville, MS, USA; ${ }^{2}$ School of \\ Veterinary Science, The University \\ of Queensland, Gatton, Queensland, \\ Australia
}

\begin{abstract}
Hypoadrenocorticism (HOAC; Addison's disease) is an endocrine condition seen in small animal practice. Dogs with this disease can present in a variety of ways from acute hypovolemic collapse to vague, chronic, waxing, and waning clinical signs. In the most common form of this disease, animals have both mineralocorticoid and glucocorticoid deficiency, resulting in hyponatremia and hyperkalemia, and signs of cortisol deficiency. The etiology may be immune-mediated destruction of the adrenal cortex, drug-induced adrenocortical necrosis (mitotane), enzyme inhibition (trilostane), or infiltrative processes such as neoplastic or fungal disease. Much less commonly, dogs have signs of cortisol deficiency, but no electrolyte changes. This is referred to as atypical HOAC. The veterinarian needs to have a clinical suspicion for HOAC to make a diagnosis in a timely manner. Treatment of dogs with an acute presentation prioritizes correcting the hypovolemia, hyperkalemia, acidosis, and hypoglycemia. Fluid therapy addresses most of these issues, but other directed therapies may be required in the most severe cases. For chronic management, all patients with Addison's disease will require replacement of glucocorticoids (usually prednisone), and most patients require replacement of mineralocorticoids with either desoxycorticosterone pivalate or fludrocortisone. Atypical Addisonians do not require mineralocorticoid supplementation, but electrolytes should be monitored in case the need arises in the future. The prognosis for dogs treated for HOAC promptly and appropriately is excellent; most patients die from other diseases. However, if the diagnosis is missed, patients may die as a consequence of HOAC. Thus, knowledge of the hallmarks of Addison's disease is imperative.
\end{abstract}

Keywords: hypoadrenocorticism, Addison's disease, adrenal

\section{Introduction}

Hypoadrenocorticism (HOAC) is an uncommon disease seen in small animal practice. Although life-threatening, it can be an extremely rewarding disease to treat, especially when the patient presents to the veterinarian in a crisis. The typical etiology is immunemediated destruction of the adrenal gland. Usually, the adrenal cortex, including the zona glomerulosa (mineralocorticoid production) and zonae fasciculata and reticularis (cortisol production), is destroyed at similar rates resulting in a lack of both cortisol and aldosterone at presentation. ${ }^{1}$ A small subset of dogs with HOAC will have normal electrolytes and low cortisol levels at presentation. If these changes persist (ie, electrolyte changes do not develop in the next few days to weeks), these animals are referred to as having atypical HOAC. The etiology of this process is not fully understood, but in some animals, it appears that the glomerulosa is spared in the immune-mediated
Correspondence: Patty Lathan Small Animal Internal Medicine, College of Veterinary Medicine, PO Box 6100, MS 39762-6100, USA

Email lathan@cvm.msstate.edu 
adrenal cortical destruction. ${ }^{2}$ Alternatively, dogs with normal electrolytes may also have subnormal aldosterone levels, suggesting immune-mediated destruction of all layers and renal compensation to maintain normal electrolytes. ${ }^{3}$ HOAC may also occur unintentionally, following the use of drugs used to treat hyperadrenocorticism. Lastly, infiltrative disease, including neoplasia and fungal disease, may affect both adrenals, resulting in adrenal insufficiency. ${ }^{4,5}$ The aim of this study was to review the diagnosis of HOAC, including naturally occurring and drug-induced, and to discuss the management of patients in crisis and long term.

\section{Signalment Typical HOAC}

Addison's disease afflicts more female dogs than male dogs, with between $64 \%$ and $70 \%$ of reported cases being female. ${ }^{6}$ The average age at presentation is 4 years although there is a wide reported age range ( 4 months to 14 years). There are several overrepresented breeds with HOAC, some with genetic predisposition. Inheritance has been shown to be autosomal recessive in Nova Scotia Duck Tolling Retrievers, Standard Poodles, and Portuguese Water Dogs and undetermined inheritance in Bearded Collies. ${ }^{7-9}$

Other breeds commonly afflicted with HOAC where inheritance has not been proven are mixed breed dogs, Poodles (other than Standard), Golden Retrievers, Cairn Terriers, Rottweilers, Great Danes, and West Highland White Terriers.

\section{Atypical HOAC}

Patients that have only cortisol deficiency are older at presentation than those with both mineralocorticoid and glucocorticoid deficiencies. Dogs with this form of disease are usually between 6 and 7 years (range $=2.75-11$ years) compared with typical dogs presenting at 4 years. ${ }^{10,11}$

The sex distribution for atypical cases is closer to $55 \%$ female; hence, more male dogs are affected compared with typical HOAC. Breeds reported include mixed breed dogs, Standard Poodles, German Shepherds, Great Danes, West Highland White Terriers, and Labrador Retrievers.

\section{latrogenic HOAC}

It is difficult to identify a specific signalment of dogs that develop unintentional iatrogenic HOAC. Mostly, they are dogs that have been treated with trilostane or mitotane; hence, they are patients being treated for hyperadrenocorticism. In papers discussing the treatment of hyperadrenocorticism, the specifics of the few patients that develop HOAC are rarely discussed, but rather as a percentage of the overall population treated; papers where adrenal necrosis was intentionally induced with mitotane to manage hyperadrenocorticism were not reviewed in this article. Most dogs with hyperadrenocorticism are 8 years or older with a mix of breeds that typically develop Cushing's disease. Details of sex are again not well documented, but female dogs are likely to be overrepresented as they are more commonly reported to develop hyperadrenocorticism. ${ }^{12,13}$

Ketoconazole has been used to treat hyperadrenocorticism in dogs. Adverse effects are reported in treated dogs, but no dogs have been reported to have a post-adrenocorticotropic hormone (ACTH) stimulated cortisol value supportive of HOAC, although the use of this drug would raise suspicion that adrenal suppression could occur. ${ }^{14}$

Additional drugs to remember other than those used to treat hyperadrenocorticism that can cause adrenal suppression include progestogens such as megestrol acetate and delmadinone..$^{15,16}$ Delmadinone acetate is used for its antiandrogen actions and is used to treat prostatic hyperplasia in intact male dogs. This drug has been reported to cause clinical HOAC and adrenal suppression for up to 14 weeks in a 2-year-old intact male Labrador Retriever. ${ }^{17}$ Long-term administration of glucocorticoids will also suppress ACTH production; therefore, abrupt discontinuation of glucocorticoids could cause signs of cortisol deficiency.

\section{History and clinical signs Typical HOAC}

The classic clinical signs are well described for Addisonian patients. Patients usually have a chronic history of gastrointestinal (GI) symptoms including inappetence, but they may also experience acute collapse with apparent sudden onset in clinical signs.

The most commonly noted clinical signs are vague and could be associated with many other disease processes: lethargy, anorexia, decreased appetite, vomiting, and weight loss. In almost $50 \%$ of patients, clinical signs are intermittent in nature.

Less commonly, animals present with symptoms of hypoglycemia that may be demonstrated as weakness, collapse, or seizures. Muscle cramps and megaesophagus have also been reported as unusual clinical signs of HOAC. ${ }^{18,19}$

\section{Atypical HOAC}

Atypical patients usually have a longer duration of clinical signs than that of the typical Addisonians. This is likely due to the fact that this form of the disease is more challenging to diagnose. Clinical signs are similarly due to the lack of 
cortisol, as in typical HOAC dogs, but hypovolemia associated with mineralocorticoid deficiency does not occur. Anorexia, lethargy, diarrhea, vomiting, and polydipsia (PD) are more prevalent in this group. ${ }^{10,11,20,21}$ Some animals may have no apparent clinical signs, but instead clinicopathological changes such as hypoalbuminemia or hypocholesterolemia, which warrant further investigation. ${ }^{20,21}$

\section{latrogenic HOAC}

Clinical signs are the same as typical HOAC cases including lethargy, vomiting, diarrhea, and occasionally collapse.

\section{Physical examination findings Typical HOAC}

Animals experiencing an Addisonian crisis may be collapsed and in shock at presentation. Weakness and lethargy are common presenting clinical signs as are varying degrees of dehydration. Other changes noted on physical examination are melena, hypothermia, abdominal pain, and slow capillary refill time. Bradycardia is also noted in $17 \%$ of dogs. ${ }^{22} \mathrm{Hypo-}$ tension is also a common finding in patients with HOAC. In one study, the mean systolic blood pressure was $90 \mathrm{mmHg}$ at presentation. ${ }^{23}$

\section{Atypical HOAC}

Signs are similar to typical cases although these patients are less likely to present in a hypovolemic crisis. Collapse may occur and has been reported secondary due to hypoglycemia, sepsis, and GI hemorrhage. ${ }^{10,11}$

\section{latrogenic HOAC}

Physical examination is expected to be the same as typical cases if the patients have electrolyte abnormalities or as the atypical patients if they do not have electrolyte abnormalities.

\section{Diagnostic test abnormalities Typical HOAC}

A complete blood count (CBC) test, serum biochemistry analysis, and urinalysis should be performed in all patients with clinical signs consistent with HOAC. Electrolyte analysis must be included in the minimum database, as sodium and potassium abnormalities are often the first specific indicators of HOAC, and electrolyte disturbances are common in patients with GI signs of any etiology and may need to be addressed during treatment. When minimal in-clinic blood work is performed without the benefit of electrolyte analysis, incorrect diagnoses can be made and HOAC may be missed. It may be easy to attribute the patients' inappetence to azotemia of renal etiology rather than endocrine.

Most dogs with HOAC are hyperkalemic and hyponatremic at diagnosis. ${ }^{1,22-24}$ Potassium concentrations usually remain $<8 \mathrm{mEq} / \mathrm{L}$ but may be as high as $10.8 \mathrm{mEq} / \mathrm{L}$. Sodium concentrations are usually between 120 and $140 \mathrm{mEq} / \mathrm{L}$ but may be as low as $107 \mathrm{mEq} / \mathrm{L} .^{22,24}$ Some dogs present with one abnormality without the other (eg, hyponatremia without hyperkalemia). Hypochloremia follows hyponatremia in about half of the patients. Metabolic acidosis is also common, due to the inability to excrete $\mathrm{H}+$ ions from the distal tubule.

Electrolyte abnormalities are not always detected early in the course of the disease and may appear as the patient's disease progresses. Emphasis is sometimes placed on the calculation of the $\mathrm{Na}+\mathrm{K}+$ ratio. The lower the sodium and the higher the potassium concentration, the lower the ratio. Although lower ratios make HOAC more likely, a high ratio does not rule it out, and dogs with low ratios do not always have HOAC. ${ }^{24}$ Therefore, the utility of the ratio is questionable. Any dog with hyponatremia and/or hyperkalemia should be considered a suspect for HOAC, regardless of the ratio.

Most patients experience some degree of azotemia, with increased blood urea nitrogen (BUN; 90\% of dogs) and creatinine $(65 \%$ of dogs $) .{ }^{5}$ This is due to a combination of decreased renal perfusion from hypovolemia and GI blood loss (increased BUN). Phosphorus is usually increased, as well. Although the azotemia is primarily prerenal in nature, most dogs with HOAC have a urine specific gravity $<1.030$ (often <1.020), which is more dilute than expected with prerenal azotemia alone. ${ }^{5}$ For this reason and the presence of hyperkalemia and hyponatremia, some Addisonians are initially misdiagnosed with acute kidney injury (AKI). Given the difference in prognosis and treatment, clinicians must consider HOAC in every suspected AKI patient, especially if the dog has a history of GI signs. Severe azotemia caused by HOAC usually resolves quickly following appropriate rehydration, and the patient's attitude also improves relatively quickly. Primary renal damage is possible with prolonged hypovolemia, but most Addisonian patients recover renal function. Conversely, patients with AKI take much longer to improve (if they ever do). In addition, significant hyperkalemia and hyponatremia rarely occur in polyuric AKI patients but often occur in anuric and oliguric AKI dogs. Addisonians may initially present with physiological oliguria (appropriately decreased urine production in the face of hypovolemia and/or dehydration). However, rehydration results in polyuria (PU). Thus, if a hypovolemic, azotemic, hyperkalemic, hyponatremic dog has a significant improvement 
in attitude and becomes polyuric upon rehydration, HOAC should be strongly considered as a differential diagnosis. Hypocholesterolemia and hypoalbuminemia occur in $\sim 10 \%$ and $15 \%$ dogs with HOAC, respectively. ${ }^{1,5}$ Both might be due to decreased hepatic production and GI malabsorption. GI blood loss exacerbates the hypoalbuminemia in some cases.

Hypoglycemia is present in about $15 \%$ of HOAC dogs, probably due to decreased gluconeogenesis and increased insulin sensitivity. It is usually subclinical but rarely can be severe enough to cause seizures. Thus, HOAC should be a differential diagnosis in adult dogs with unexplained hypoglycemia. ${ }^{1}$

Total and ionized hypercalcemia occurs in about 30\% of Addisonians. It is not usually the presenting complaint, but HOAC should be considered a differential diagnosis in hypercalcemic patients. ${ }^{1}$

Increased alanine aminotransferase, alkaline phosphatase, and aspartate aminotransferase are also frequently seen (20\%-30\%) in HOAC dogs, possibly secondary to hepatic hypoperfusion and cholestasis. ${ }^{1}$

At diagnosis, about $30 \%$ of Addisonian dogs are anemic. This increases to $70 \%$ following rehydration. ${ }^{1,5}$ It is usually a mild normochromic, normocytic, nonregenerative anemia, and GI blood loss should be suspected in patients with severe anemia. The lack of a stress leukogram (neutrophilia, lymphocytosis, monocytosis, and eosinopenia) in a sick dog is suggestive of HOAC. Occasionally, lymphocytosis and eosinophilia occur $(10 \%)$, but neutropenia is rare.

Diagnostic imaging is not necessary to diagnose HOAC in most patients, but abdominal imaging is often performed in the initial workup of patients with GI signs. Imaging is recommended, however, in Addisonian patients that do not respond to appropriate therapy. These patients may have concurrent disease or HOAC due to infiltrative disease (eg, neoplasia or fungal disease invading the adrenal cortex). In these patients, the adrenal gland may appear normal or larger in size; this should raise suspicion of infiltrative disease.

In patients with primary idiopathic HOAC, abdominal radiographs and ultrasound may reveal a small liver, consistent with hypovolemia. ${ }^{25}$ Dogs with symptoms similar to HOAC that have thoracic radiographs often reveal nonspecific signs of hypovolemia, including microcardia. Megaesophagus is seen infrequently. Adrenal gland length and thickness might be smaller than in normal dogs, when measured on abdominal ultrasound, but the overlap in adrenal size between normal and HOAC patients does not allow definitive differentiation. In a study looking at adrenal measurements, 28 of 29 dogs with confirmed HOAC had a left adrenal thickness of $<3.2 \mathrm{~mm}$. Two of $10 \mathrm{dogs}$ with nonadrenal causes of hyperkalemia and hyponatremia had adrenals that measured $<3.2 \mathrm{~mm}$, but none of the normal dogs had adrenals this small. ${ }^{26}$ Right adrenals in this study were not always identified and had more variation than the left. A left adrenal gland measuring $<3.2 \mathrm{~mm}$ would be further support of HOAC.

\section{Atypical HOAC}

Biochemical abnormalities may be similar to typical HOAC cases, although by definition the sodium and potassium are within the normal limits. The lack of the sodium and potassium changes may result in the clinician overlooking HOAC as a possible diagnosis since this is the distinguishing biochemical change. Other changes that might alert the veterinarian to consider this disease are hypocholesterolemia (mean $94 \mathrm{mg} / \mathrm{dL}$ or $2.4 \mathrm{mmol} / \mathrm{L}$ ) and hypoalbuminemia $(2.1 \mathrm{~g} / \mathrm{dL}$ or $21 \mathrm{~g} / \mathrm{L})$. These abnormalities are more marked in atypical than in typical cases.

Anemia is more prevalent in this group than in typical HOAC cases in one study with an average hematocrit of $31 \%$ compared with $39 \%$ in typical cases. ${ }^{10}$ In atypical cases, the anemia may be due to GI hemorrhage or anemia of chronic disease. Changes in white cell counts are similar to typical cases.

Thoracic radiographs may reveal megaesophagus. Hypovolemia is very unlikely in dogs with cortisol deficiency, but changes consistent with low blood volume may be present.

In a recent study, adrenal imaging was performed in 15 dogs with atypical HOAC; $56 \%$ of them had a left adrenal gland measuring $<3.2 \mathrm{~mm}$, although adrenal thickness ranged from 1.1 to $5.1 \mathrm{~mm}$. In two dogs, neither adrenal could be identified. $^{21}$

\section{latrogenic HOAC}

Biochemical and $\mathrm{CBC}$ changes are similar to those of typical cases; there are no additional distinguishing features noted in this group. Adrenal size, when assessed ultrasonographically, may be larger in dogs treated with trilostane. Dogs with hyperadrenocorticism treated with trilostane initially had adrenal thickness $>5 \mathrm{~mm}$; within 6 weeks of initiating therapy, adrenals had up to $60 \%$ increase in thickness; the median was $21 \%$. There may also be a noticeable change in adrenal appearance with a hypoechoic outer zone and a hyperechoic center. ${ }^{27}$

\section{Specific adrenal testing Typical HOAC}

\section{Endocrine diagnostics}

An ACTH stimulation test is required for a definitive diagnosis of HOAC. A baseline cortisol concentration alone can 
help rule out the diagnosis of HOAC, but cannot be used to definitely diagnose it. ${ }^{28}$

During an ACTH stimulation test, a supraphysiological dose of synthetic ACTH is administered, and the cortisol concentration is measured 1-2 hours later. In a dog with normally functioning adrenal glands, ACTH should cause a significant increase in cortisol concentration. In HOAC dogs, minimal to no increase in cortisol will occur. Note that there are both short-acting and depot forms of tetracosactrin; both may be used to diagnose HOAC, but there are variations in protocol, as detailed below.

To perform an ACTH stimulation test, a baseline serum sample is first obtained. Then, $5 \mathrm{ug} / \mathrm{kg}$ (up to $250 \mathrm{ug} / \mathrm{dog}$ ) of synthetic ACTH (cosyntropin or tetracosactrin) is administered intravenously (IV). ${ }^{29}$ One hour later, another serum sample is obtained. Cortisol concentration is then measured in both pre- and post-ACTH serum samples. A depot formulation of tetracosactrin has been investigated for its utility in assessing adrenal function due to periods of limited availability of the shorter-acting form. The depot preparation may be used to diagnose $\mathrm{HOAC}$ at $5 \mathrm{ug} / \mathrm{kg}$, but due to its formulation it should be administered intramuscularly only (hence it is less desirable to use on hypovolemic patients). The timing of peak cortisol concentrations was variable between patients, but a serum sample taken 1 hour after depot ACTH administration was considered adequate to assess for HOAC. ${ }^{30}$

Protocols for the ACTH stimulation test vary when testing for HOAC or hyperadrenocorticism. The use of compounded ACTH gel is not recommended for the diagnosis of HOAC, due to inconsistent results. ${ }^{31}$ In addition, since dogs with HOAC are often hypovolemic, intramuscular administration of $\mathrm{ACTH}$ is not recommended in unstable patients.

The diagnosis of HOAC requires a post-ACTH cortisol concentration $<2 \mathrm{ug} / \mathrm{dL}(55 \mathrm{nmol} / \mathrm{L})$; a post-ACTH cortisol concentration $>2 \mathrm{ug} / \mathrm{dL}$ rules out the diagnosis of HOAC. Note that this test does not differentiate between primary, secondary, and iatrogenic Addison's disease. However, iatrogenic HOAC, due to the administration of drugs that suppress adrenal function such as long-term use of glucocorticoids, should be identified based on historical findings.

The administration of glucocorticoids prior to an ACTH stimulation test can interfere with and/or cause decreased response to the test. Most synthetic glucocorticoids, including prednisone and methylprednisolone, cross-react with the cortisol assay, which can cause falsely increased results. Dexamethasone and triamcinolone do not. Thus, if a steroid is given just prior to the test, dexamethasone is recommended. Other short-acting glucocorticoids (such as prednisone) should be withheld for 12-24 hours prior to the test.
Longer-acting glucocorticoids, such as methylprednisolone acetate, should be withheld for at least 4 weeks prior to the test to avoid cross-reactivity.

The administration of glucocorticoids decreases endogenous ACTH concentrations, which causes atrophy of the adrenal cortex (fasciculata and reticularis). A blunted response to the ACTH stimulation test is expected in patients that have received glucocorticoids (oral, parenteral, or topical) within about a month of the test or longer in case of longer-acting glucocorticoids (such as methylprednisolone acetate). A poststimulation cortisol between 2 and $5 \mathrm{ug} / \mathrm{dL}$ is often the result of recent steroid administration, not HOAC.

One way to save money with ACTH stimulation tests is to freeze the unused portion of a vial of cosyntropin. Cosyntropin can be frozen in plastic syringes (not in glass, as it binds to ACTH) for up to 6 months. ${ }^{32}$ The author freezes $50 \mathrm{ug}$ aliquots per syringe (enough for the $10 \mathrm{~kg}$ patient) and does not refreeze any extra that has been thawed. The use of a frost-free freezer is not recommended, as the effect of the freeze/thaw cycles on the ACTH is unknown.

A baseline cortisol concentration is a less expensive way to rule out HOAC. Since the cortisol concentration in almost any patient with $\mathrm{HOAC}$ is $<2 \mathrm{ug} / \mathrm{dL}(55 \mathrm{nmol} / \mathrm{L})$, a baseline cortisol concentration of $>2 \mathrm{ug} / \mathrm{dL}(55 \mathrm{nmol} / \mathrm{L})$ rules out the diagnosis. This test has a $99.3 \%$ specificity, but only a $75 \%$ sensitivity in animals with abnormal electrolytes. ${ }^{33}$ Hence, if the patient has a baseline cortisol concentration $<2 \mathrm{ug} / \mathrm{dL}$ (55 nmol/L), an ACTH stimulation test is required for a definitive diagnosis of HOAC.

Pre- and post-ACTH stimulated aldosterone levels are low or undetectable in most dogs with HOAC. ${ }^{3}$ This is to be expected as the process of immune destruction of the adrenal gland effects, both the zona glomerulosa and fasciculata, and most patients have electrolyte changes consistent with aldosterone deficiency.

For the definitive diagnosis of primary HOAC, an alternative to the ACTH stimulation test is the cortisol-to-ACTH ratio (CAR). Since dogs with HOAC have low cortisol concentrations and high ACTH concentrations (due to the lack of negative feedback), the CAR is significantly lower in patients with HOAC than in normal dogs or dogs with nonadrenal illness. ${ }^{34,35}$ The benefit of the CAR is that, if collected and handled properly, a single blood sample can be used to definitively diagnose HOAC, without the need for synthetic ACTH. The disadvantage is that it requires the measurement of $\mathrm{ACTH}$, which degrades quickly and requires specialized sample handling. Laboratory recommendations vary, but most recommend freezing the sample immediately after collection and keeping it frozen while shipping or the 
addition of aprotinin to the sample. If the ACTH degrades prior to measurement, the ratio will be falsely increased, and the diagnosis of Addison's disease could be missed. In addition, the CAR cannot be used for the diagnosis of secondary HOAC. Two recent studies showed that the evaluation of the CAR differentiates patients with HOAC from patients with signs mimicking HOAC, but found to have other diseases. ${ }^{34,35}$ However, the ranges of CARs differed significantly between studies; therefore, more investigation is necessary prior to using the CAR clinically.

\section{Atypical HOAC}

Animals with normal electrolytes will have the same poor response to an ACTH stimulation test as typical HOAC dogs. The sensitivity and specificity of resting cortisol are different for animals that have normal electrolytes, $100 \%$ and $65 \%$ respectively, reinforcing that an ACTH stimulation test is required to confirm the diagnosis of HOAC and the specificity is poor. ${ }^{33}$

Aldosterone levels are not commonly reported for atypically presenting dogs. In a study that measured aldosterone levels in 70 Addisonian dogs, 3 had normal electrolytes and one had normal sodium with hypokalaemia. ${ }^{3}$ Aldosterone was low in all 4 dogs. It is thought that renal compensation mechanisms in these dogs were maintaining normal electrolytes. One dog developed electrolyte changes after 6 months, and another dog had normal electrolytes for 2 years. The other two dogs were started on mineralocorticoid supplementation.

\section{latrogenic HOAC}

Animals being treated with medication to control signs of hyperadrenocorticism intentionally have a subnormal post-ACTH cortisol with the range aimed to be $>2 \mathrm{ug} / \mathrm{dL}$ $(55 \mathrm{nmol} / \mathrm{L})$ and $<7 \mathrm{ug} / \mathrm{dL}(200 \mathrm{nmol} / \mathrm{L})$. Some apparently well-controlled animals with no clinical signs of HOAC will have a post-ACTH stimulation cortisol $<2 \mathrm{ug} / \mathrm{dL}(55 \mathrm{nmol} / \mathrm{L})$. A dose reduction of trilostane or mitotane is recommended as these animals are at an increased risk of developing clinical HOAC. If the patient has a post-ACTH stimulation $<2 \mathrm{ug} / \mathrm{dL}$ ( $55 \mathrm{nmol} / \mathrm{L})$ and clinical signs and biochemical changes are consistent with Addison's disease, then HOAC should be suspected.

\section{Therapy}

\section{Typical and iatrogenic HOAC}

\section{Acute management}

Presentations of HOAC fall along a continuum of disease. While some patients present in hypovolemic shock with severe hyperkalemia and hypokalemia (due to aldosterone deficiency), most are less critical but need IV fluids and other supportive therapy. The most severe cases (true Addisonian crises) will be discussed in this section, but some of the therapies apply to dogs on the less severe end of the continuum.

The initial goals of treatment of Addisonian crisis are to correct hypovolemia, hypotension, hyperkalemia and associated arrhythmias, hypoglycemia, and acidosis. Aggressive IV fluid resuscitation is the top priority. ${ }^{36}$ In addition to correcting hypovolemia, it also helps treat hyperkalemia, hyponatremia, hypochloremia, and acidosis. One third of a shock bolus of crystalloids $(20-30 \mathrm{~mL} / \mathrm{kg}$ ) is given initially, and response is assessed based on heart rate, pulse quality/ blood pressure, capillary refill time, and mental status. Additional fluids are then given as necessary.

Historically, $0.9 \% \mathrm{NaCl}$ has been recommended as the fluid of choice, since it contains sodium and chloride, but no potassium. $^{22,36,37}$ However, Normosol-R ${ }^{\circledR}$, Plasma-Lyte $A^{\circledR}$, and/or lactated Ringer's solution (LRS) are preferred by some clinicians, since they are more alkalinizing than saline, thus correcting acidosis more efficiently. Although they contain a small amount of potassium, these crystalloids still allow for the correction of hyperkalemia via dilution and increased renal perfusion. ${ }^{38}$ Hydroxyethyl starch can also be used in conjunction with crystalloids for more rapid fluid resuscitation and in patients with hypoalbuminemia, at a dosage of $5-10 \mathrm{~mL} / \mathrm{kg}$.

Sodium concentration should not be increased by $>0.5 \mathrm{mEq} / \mathrm{kg} / \mathrm{h}$, as myelinolysis may occur with more rapid correction. ${ }^{39}$ Thus, hypertonic saline should not be used to treat a known Addisonian crisis. Normosol-R, Plasma-Lyte $A$, and LRS all have lower sodium concentrations than $0.9 \%$ saline (140,140,130, and $154 \mathrm{mEq} / \mathrm{L}$, respectively). Their use is sometimes preferred in patients with severe hyponatremia, as they are less likely to increase the serum sodium concentration as rapidly as $0.9 \%$ saline.

Hyperkalemia is often the most acutely life-threatening consequence of HOAC and must be addressed immediately. Mild-to-moderate hyperkalemia usually responds to fluid therapy alone. However, severe hyperkalemia (>8-9 mEq/L) and/or hyperkalemia accompanied by life-threatening arrhythmias (severe bradycardia, absent P-waves, and idioventricular rhythm) requires specific therapy.

Calcium gluconate is given for its cardioprotective effects, which are rapid (within minutes and last about an hour), and will often maintain the patient's life while allowing time for fluids and other therapies to take effect. ${ }^{40}$ However, it does not directly decrease the potassium concentration; $0.5-1.0 \mathrm{~mL} / \mathrm{kg}$ 
of $10 \%$ calcium gluconate is given slowly over 15 minutes, while monitoring the electrocardiogram (ECG)..$^{22,36,41}$ Rapid administration may cause additional arrhythmias, such as shortened QT interval and more severe bradycardia. Note that calcium chloride is not recommended in dogs due to venous irritation.

IV regular insulin helps drive potassium intracellularly; $0.2-0.5 \mathrm{U} / \mathrm{kg}$ regular insulin is given IV, followed by $1-2 \mathrm{~g}$ of dextrose (diluted to $25 \%$ in an isotonic crystalloid) per unit of insulin administered. Dextrose should then be added to the IV fluids (eg, $0.9 \%$ saline or LRS) to produce a $1.25 \%-2.5 \%$ dextrose solution, and blood glucose should be monitored every 30-60 minutes until it stabilizes within the reference range; $5 \%$ dextrose alone should not be given, as it becomes hypotonic and enters the interstitial space once the dextrose is metabolized. The combination of insulin and dextrose takes effect quickly and lasts $\sim 15-30$ minutes. ${ }^{38}$

Hypoglycemia is uncommon but should be treated with an IV dose of $1 \mathrm{~mL} / \mathrm{kg}$ of $25 \%$ dextrose (a 1:1 solution of $50 \%$ dextrose and $0.9 \%$ saline) if identified. Maintenance fluids should then be supplemented with dextrose to make a $1.25 \%-5 \%$ dextrose solution.

Glucocorticoid deficiency is responsible for the hypoglycemia, GI signs, and general debility of patients in crisis and may also contribute to hypotension. Supplementation should be instituted after the life-threatening issues (hypovolemia, hyperkalemia, and hypoglycemia) have been initially addressed. Dexamethasone sodium phosphate $(0.25 \mathrm{mg} / \mathrm{kg} \mathrm{IV})$ is recommended, since it does not cross-react with the cortisol assay and can be given prior to the ACTH stimulation test. Since it has $\sim 8$ times the glucocorticoid activity of prednisone, this is equivalent to $2 \mathrm{mg} / \mathrm{kg}$ of prednisone or 10 times the physiological dose. ${ }^{41}$ Higher doses previously recommended (such as $2 \mathrm{mg} / \mathrm{kg}$ ) are excessive and generally unnecessary. Hydrocortisone sodium succinate $(0.5-0.625 \mathrm{mg} / \mathrm{kg} / \mathrm{h})$ and prednisolone sodium succinate $(2 \mathrm{mg} / \mathrm{kg}$ ) can also be used. Hydrocortisone has the added benefit of mineralocorticoid activity that, according to one recent study, may decrease the amount of fluid resuscitation required in some patients. ${ }^{42}$ However, since hydrocortisone and prednisone cross-react with the cortisol assay, they should not be given until after the ACTH stimulation test.

Additional supportive therapy is provided as necessary. Dogs with GI signs are given gastroprotectants, including proton pump inhibitors (omeprazole or pantoprazole, which can be given IV) and sucralfate. Due to potential bacterial translocation from the GI tract, dogs with significant GI signs may be given prophylactic antibiotics (such as ampicillin), but this is controversial. Packed red cell or whole blood transfusion is occasionally necessary in dogs with severe GI blood loss.

Frequent reassessment of patients is necessary following initial stabilization. Fluid rates should be adjusted to correct dehydration and azotemia. ECG should be reassessed until arrhythmias resolve. Electrolyte concentrations should be rechecked following initial stabilization and then every 6-12 hours until potassium and sodium concentrations normalize. Sodium concentration should not be corrected by $>12 \mathrm{mEq} / \mathrm{L}$ per 24-hour period.

IV fluids are continued until the dog is able to eat and maintain hydration on her own, and mineralocorticoid supplementation (fludrocortisone or desoxycorticosterone pivalate $[\mathrm{DOCP}])$ is provided following a definitive diagnosis of HOAC. Dexamethasone should be continued at a dose of $0.15-0.2 \mathrm{mg} / \mathrm{kg} / \mathrm{d}$ until prednisone can be given orally. Prednisone is then started at $0.5 \mathrm{mg} / \mathrm{kg}$ twice a day. Following discharge of the patient, prednisone is usually decreased to $\sim 0.5 \mathrm{mg} / \mathrm{kg}$ per day and then decreased further based on clinical signs, as discussed in chronic management.

Most patients in Addisonian crisis respond quickly to treatment (within hours), although the more severely debilitated ones take 2-3 days to see dramatic improvement. Dogs are usually discharged 3-5 days after initial presentation. ${ }^{22}$

\section{Chronic management}

Chronic therapy for HOAC consists of lifelong glucocorticoid and mineralocorticoid supplementation to replace the missing cortisol and aldosterone.

Mineralocorticoids are given to correct hyperkalemia, hyponatremia, and hypochloremia. Either a monthly injection (DOCP) or a twice daily pill (fludrocortisone) can be used. However, a 2014 study demonstrated that DOCP suppresses plasma renin activity (PRA) more effectively than fludrocortisone. ${ }^{43}$ Since PRA is increased in untreated Addisonian dogs due to aldosterone deficiency, this suggests that DOCP is more effective than fludrocortisone at replacing aldosterone in Addisonians. DOCP can be given subcutaneously or intramuscularly. ${ }^{44}$ Although the labeled dose is $2.2 \mathrm{mg} / \mathrm{kg}$ (Zycortal $^{\circledR}$, Dechra Pharmaceuticals, Overland Park, KS, USA; Percorten- $\mathrm{V}^{\circledR}$, Elanco, Indianapolis, IN, USA), studies have proven that lower doses can be effective. In a publication from 1997, the final median dose was $1.69 \mathrm{mg} / \mathrm{kg}$, and only $18 \%$ of the dogs required the labeled dose of $2.2 \mathrm{mg} / \mathrm{kg} .{ }^{45}$ In a more recent publication, over half of the dogs received $\leq 1.5 \mathrm{mg} / \mathrm{kg}$, with a median final dose of $1.3 \mathrm{mg} / \mathrm{kg} / \mathrm{dose} .^{46}$ Due to client concerns about the expense of the medication, 
we (PL and AT) routinely begin patients on a dose of $1.5 \mathrm{mg} /$ $\mathrm{kg}$ (or less for larger dogs). As in the 2013 study, we have not seen any severe clinical signs when using this dose, but occasional patients require a dose closer to the label dose. ${ }^{46}$

DOCP is initially given every 25 days. Electrolyte concentrations are measured 2 weeks and then 25 days, following the first injection. The 14-day measurement helps determine whether the dose is correct, whereas the 25-day recheck confirms that the interval is acceptable. If hyperkalemia and/or hyponatremia is present, the dose (14-day recheck) is increased by $10 \%-15 \%$, or the interval is decreased by 2 days (25-day recheck). If hypokalemia and/or hypernatremia is present, the dose (14-day recheck) is decreased by $10 \%-15 \%$. We usually increase the dosing interval by 2-3 days each month, measuring the electrolytes before each injection, to ensure that they are within the reference range, until the interval reaches 30-31 days, for owner convenience. After adjusting the interval, if additional dose decreases are requested for financial reasons, the dose can be decreased by $\sim 10 \%$ per month, measuring electrolytes immediately prior to each injection to ensure that the electrolyte concentrations are still acceptable. The author rarely decreases below $<1 \mathrm{mg} / \mathrm{kg} / \mathrm{month}$. Clients must understand that using lower doses leaves less room for error and requires tight adherence to the dosing schedule. They must be warned not to prolong the dosing interval to save money without veterinary advice; this often results in Addisonian crisis, which is significantly more expensive to treat than a single injection of DOCP and is life-threatening.

Following stabilization of DOCP dose, electrolytes should be measured every 3-6 months. Dogs that are receiving DOCP injections must also receive a glucocorticoid (usually prednisone), as DOCP has no glucocorticoid activity. Many owners can be taught to give the injection at home after the dose and timing of injection have been established.

Side effects of DOCP are uncommon, but PU/PD has been reported. This is usually due to concurrent prednisone administration..$^{45}$ However, PU/PD is occasionally reported to be worse immediately following DOCP injection and to improve throughout the month. In these cases, prolonging the dose interval or decreasing the dose, while monitoring electrolytes, may result in improvement.

Fludrocortisone is a shorter-acting oral mineralocorticoid that is available in $0.1-\mathrm{mg}$ tablets. The starting dose is $0.01-0.02 \mathrm{mg} / \mathrm{kg} / \mathrm{day}$. Although once-daily dosing is effective in some dogs, 12 of 37 Addisonians had persistent hyperkalemia and/or hyponatremia until they were switched to twice-daily administration $(0.01-0.02 \mathrm{mg} / \mathrm{kg} /$ day divided $) .{ }^{47}$
Electrolytes are measured in 1-week increments until the electrolytes are within the reference range. If hyponatremia and/or hyperkalemia is present, the dose is increased by $0.05-0.1 \mathrm{mg} /$ day. If hypernatremia and/or hypokalemia is present, the dose is decreased. Once the dose has normalized, electrolyte concentrations are rechecked every 3-6 months. The dose often increases during the first 1-2 years of therapy. Since fludrocortisone has some glucocorticoid activity, not all treated dogs need additional glucocorticoids in the long term. However, all dogs should be given glucocorticoids (such as prednisone) initially and until the electrolytes and clinical signs have normalized and then tapered as described below. ${ }^{46}$

$\mathrm{PU}, \mathrm{PD}$, polyphagia, and panting are the most commonly reported side effects of fludrocortisone therapy. Most of these are due to the glucocorticoid properties of fludrocortisone and resolve when the prednisone dose is tapered and/or the patient is switched to DOCP. ${ }^{45}$

Glucocorticoids are used to control the nonspecific and GI signs associated with HOAC, and prednisone (or prednisolone) is the most frequently used. All dogs receiving DOCP need glucocorticoid supplementation, and about 50\% of dogs given fludrocortisone will need additional glucocorticoid supplementation in the long term. The physiological dose of prednisone is $0.1-0.25 \mathrm{mg} / \mathrm{kg} / \mathrm{d}$, although some dogs (particularly larger breeds) do well on $0.05 \mathrm{mg} / \mathrm{kg} / \mathrm{d}$. Following diagnosis, the patient is usually sent home on a dose of $0.5 \mathrm{mg} / \mathrm{kg} /$ day and decreased over the following several weeks. The dose is tapered based on the clinical signs and side effects. If the dog has any GI sign or is not acting themself, the dose is increased. If PU/PD, polyphagia, or panting occurs, the dose needs to be decreased. Remember that the dose is adjusted based on clinical signs and that an ACTH stimulation test is never repeated in patients with primary HOAC.

During times of stress, the prednisone dose is usually doubled. ${ }^{38}$ "Stressful" situations include physiological and emotional stress, such as when a dog goes to the vet clinic for electrolyte rechecks, when new visitors are in the household, when the dog goes hunting (if applicable), during boarding, and when the dog has a concurrent illness. The increased dose is usually started in the morning before the stress begins and continued for a day or two following the end of the stressful event. Astute clients are usually able to identify when their dog needs additional prednisone and adjust the dose appropriately. However, some clients need frequent reminders, particularly when they present for electrolyte evaluation. 


\section{Atypical HOAC}

Therapy for atypical HOAC consists of replacement of cortisol, usually with prednisone, given at $0.1-0.25 \mathrm{mg} / \mathrm{kg} / \mathrm{day}$, as directed for patients with classic HOAC. The goal is to give enough prednisone to control the clinical signs of HOAC, while not causing side effects of prednisone administration. Additional prednisone (twice normal) is recommended during times of stress.

Dogs with atypical HOAC sometimes develop signs of mineralocorticoid deficiency (electrolyte abnormalities) weeks to months after the initial diagnosis (usually within 1 year). It is impossible to predict which dogs will develop electrolyte abnormalities; therefore, reevaluation of the electrolytes is recommended at 1 and 3 months following initial diagnosis and then every 6 months thereafter.

\section{Prognosis (typical and atypical Addison's disease)}

With appropriate treatment and monitoring, the prognosis for dogs with naturally occurring HOAC is excellent. Dogs enjoy a good quality of life and may participate in normal activities, as long as they are provided with additional prednisone as needed. Median survival time in dogs treated between 1979 and 1993 was 4.7 years, and most dogs died from causes other than Addison's disease. ${ }^{45}$ Consistent medication and patient monitoring must be stressed to owners, as skipping medication or prolonging the treatment interval for DOCP, without veterinary approval, may lead to Addisonian crisis.

\section{Disclosure}

The authors report no conflicts of interest in this work.

\section{References}

1. Peterson ME, Kintzer PP, Kass PH. Pretreatment clinical and laboratory findings in dogs with hypoadrenocorticism: 225 cases (1979-1993). $J$ Am Vet Med Assoc. 1996;208(1):85-91.

2. Frank CB, Valentin SY, Scott-Moncrieff JC, Miller MA. Correlation of inflammation with adrenocortical atrophy in canine adrenalitis. J Comp Pathol. 2013;149(2-3):268-279.

3. Baumstark ME, Sieber-Ruckstuhl NS, Muller C, Wenger M, Boretti FS, Reusch CE. Evaluation of aldosterone concentrations in dogs with hypoadrenocorticism. J Vet Intern Med. 2014;28(1):154-159.

4. Kook PH, Grest P, Raute-Kreinsen U, Leo C, Reusch CE. Addison's disease due to bilateral adrenal malignancy in a dog. J Small Anim Pract. 2010;51(6):333-336.

5. Feldman EC, Nelson RW. Canine and Feline Endocrinology and Reproduction. 3rd ed. St. Louis, MO: Saunders; 2004.

6. Hanson JM, Tengvall K, Bonnett BN, Hedhammar Å. Naturally occurring adrenocortical insufficiency - an epidemiological study based on a Swedish-insured dog population of 525,028 dogs. J Vet Intern Med. 2016;30(1):76-84.

7. Hughes AM, Nelson RW, Famula TR, Bannasch DL. Clinical features and heritability of hypoadrenocorticism in Nova Scotia Duck Tolling Retrievers: 25 cases (1994-2006). J Am Vet Med Assoc. 2007;231(3):407-412.
8. Famula TR, Belanger JM, Oberbauer AM. Heritability and complex segregation analysis of hypoadrenocorticism in the standard poodle. $J$ Small Anim Pract. 2003;44(1):8-12.

9. Oberbauer AM, Benemann KS, Belanger JM, Wagner DR, Ward JH, Famula TR. Inheritance of hypoadrenocorticism in bearded collies. Am J Vet Res. 2002;63(5):643-647.

10. Thompson AL, Scott-Moncrieff JC, Anderson JD. Comparison of classic hypoadrenocorticism with glucocorticoid-deficient hypoadrenocorticism in dogs: 46 cases (1985-2005). J Am Vet Med Assoc. 2007;230(8):1190-1194.

11. Lifton SJ, King LG, Zerbe CA. Glucocorticoid deficient hypoadrenocorticism in dogs: 18 cases (1986-1995). J Am Vet Med Assoc. 1996;209(12):2076-2081.

12. Braddock JA, Church DB, Robertson ID, Watson AD. Trilostane treatment in dogs with pituitary-dependent hyperadrenocorticism. Aust Vet J. 2003;81(10):600-607.

13. Alenza DP, Arenas C, Lopez ML, Melian C. Long-term efficacy of trilostane administered twice daily in dogs with pituitary-dependent hyperadrenocorticism. J Am Anim Hosp Assoc. 2006;42(4): 269-276.

14. Lien YH, Huang HP. Use of ketoconazole to treat dogs with pituitarydependent hyperadrenocorticism: 48 cases (1994-2007). J Am Vet Med Assoc. 2008;233(12):1896-1901.

15. van den Broek AHM, O'Farrell V. Suppression of adrenocortical function in dogs receiving therapeutic doses of megestrol acetate. J Small Anim Pract. 1994;35(6):285-288.

16. Court EA, Watson AD, Church DB, Emslie DR. Effects of delmadinone acetate on pituitary-adrenal function, glucose tolerance and growth hormone in male dogs. Aust Vet J. 1998;76(8):555-560

17. Kempker K, Gussow A, Neiger R. Transienter, sekundärer Hypoadrenokortizismus nach Behandlung mit Delmadinonacetat (Tardastrex ${ }^{\circledR}$ ) bei einem zwei Jahre alten Rüden [Transient, secondary hypoadrenocorticism after treatment with delmadinone acetate (Tardastrex ${ }^{\circledR}$ ) in a two year old male dog]. Schweiz Archiv Tierheilkd. 2016;158(3):193-197. German.

18. Saito M, Olby NJ, Obledo L, Gookin JL. Muscle cramps in two standard poodles with hypoadrenocorticism. J Am Anim Hosp Assoc. 2002;38(5):437-443.

19. Bartges JW, Nielson DL. Reversible megaesophagus associated with atypical primary hypoadrenocorticism in a dog. J Am Vet Med Assoc. 1992;201(6):889-891.

20. Sadek D, Schaer M. Atypical Addison's disease in the dog: a retrospective survey of 14 cases. J Am Anim Hosp Assoc. 1996;32(2):159-163.

21. Wakayama JA, Furrow E, Merkel LK, Armstrong PJ. A retrospective study of dogs with atypical hypoadrenocorticism: a diagnostic cut-off or continuum? J Small Anim Pract. 2017;58:365-371.

22. Melián C, Peterson ME. Diagnosis and treatment of naturally occurring hypoadrenocorticism in 42 dogs. J Small Anim Pract. 1996;37: 268-275.

23. Seth M, Drobatz KJ, Church DB, Hess RS. White blood cell count and the sodium to potassium ratio to screen for hypoadrenocorticism in dogs. J Vet Intern Med. 2011;25(6):1351-1356.

24. Adler JA, Drobatz KJ, Hess RS. Abnormalities of serum electrolyte concentrations in dogs with hypoadrenocorticism. J Vet Intern Med. 2007;21(6):1168-1173.

25. Melian C, Stefanacci J, Peterson ME, Kintzer PP. Radiographic findings in dogs with naturally-occurring primary hypoadrenocorticism. J Am Anim Hosp Assoc. 1999;35(3):208-212.

26. Wenger M, Mueller C, Kook PH, Reusch CE. Ultrasonographic evaluation of adrenal glands in dogs with primary hypoadrenocorticism or mimicking diseases. Vet Rec. 2010;167(6):207-210.

27. Ruckstuhl NS, Nett CS, Reusch CE. Results of clinical examinations, laboratory tests, and ultrasonography in dogs with pituitarydependent hyperadrenocorticism treated with trilostane. Am JVet Res. 2002;63(4):506-512.

28. Lennon EM, Boyle TE, Hutchins RG, et al. Use of basal serum or plasma cortisol concentrations to rule out a diagnosis of hypoadrenocorticism in dogs: 123 cases (2000-2005). JAm Vet Med Assoc. 2007;231(3):413-416. 
29. Lathan P, Moore GE, Zambon S, Scott-Moncrieff JC. Use of a low-dose ACTH stimulation test for diagnosis of hypoadrenocorticism in dogs. J Vet Intern Med. 2008;22(4):1070-1073.

30. Sieber-Ruckstuhl N, Burkhardt W, Hofer-Inteeworn N, et al. Cortisol response in healthy and diseased dogs after stimulation with a depot formulation of synthetic ACTH. JVet Intern Med. 2015;29(6):1541-1546.

31. Hill K, Scott-Moncrieff JC, Moore G. ACTH stimulation testing: a review and a study comparing synthetic and compounded ACTH products. Vet Med. 2004;99(2):134-147.

32. Frank LA, Oliver JW. Comparison of serum cortisol concentrations in clinically normal dogs after administration of freshly reconstituted versus reconstituted and stored frozen cosyntropin. JAm Vet Med Assoc. 1998;212(10):1569-1571.

33. Gold AJ, Langlois DK, Refsal KR. Evaluation of basal serum or plasma cortisol concentrations for the diagnosis of hypoadrenocorticism in dogs. J Vet Intern Med. 2016;30(6):1798-1805.

34. Lathan P, Scott-Moncrieff JC, Wills RW. Use of the cortisol-to-ACTH ratio for diagnosis of primary hypoadrenocorticism in dogs. JVet Intern Med. 2014;28(5):1546-1550.

35. Boretti FS, Meyer F, Burkhardt WA, et al. Evaluation of the cortisolto-ACTH ratio in dogs with hypoadrenocorticism, dogs with diseases mimicking hypoadrenocorticism and in healthy dogs. JVet Intern Med. 2015;29(5):1335-1341.

36. Schaer M. The treatment of acute adrenocortical insufficiency in the dog. J Vet Emerg Crit Care. 2001;11(1):7-14.

37. Ettinger SJ, Feldman EC, editors. Textbook of Veterinary Internal Medicine: Diseases of the Dog and the Cat. St. Louis, MO: Elsevier; 2017.
38. Feldman EC, Nelson RW, Reusch C, Scott-Moncrieff JC. Canine and Feline Endocrinology. 4th ed. London: Elsevier Health Sciences; 2014.

39. Brady CA, Vite CH, Drobatz KJ. Severe neurologic sequelae in a dog after treatment of hypoadrenal crisis. J Am Vet Med Assoc. 1999;215(2):222-225, 210.

40. DiBartola SP. Fluid, Electrolyte, and Acid-Base Disorders in Small Animal Practice. 4th ed. St. Louis, MO: Saunders/Elsevier; 2012.

41. Plumb DC. Veterinary Drug Handbook. 4th ed. ed. Ames, IA: Iowa State Press; 2002.

42. Gunn E, Shiel RE, Mooney CT. Hydrocortisone in the management of acute hypoadrenocorticism in dogs: a retrospective series of 30 cases. J Small Anim Pract. 2016;57(5):227-233.

43. Baumstark ME, Nussberger J, Boretti FS, et al. Use of plasma renin activity to monitor mineralocorticoid treatment in dogs with primary hypoadrenocorticism: desoxycorticosterone versus fludrocortisone. $J$ Vet Intern Med. 2014;28(5):1471-1478.

44. McCabe MD, Feldman EC, Lynn RC, Kass PH. Subcutaneous administration of desoxycorticosterone pivalate for the treatment of canine hypoadrenocorticism. J Am Anim Hosp Assoc. 1995;31(2):151-155.

45. Kintzer PP, Peterson ME. Treatment and long-term follow-up of 205 dogs with hypoadrenocorticism. J Vet Intern Med. 1997;11(2): 43-49.

46. Bates JA, Shott S, Schall WD. Lower initial dose desoxycorticosterone pivalate for treatment of canine primary hypoadrenocorticism. Aust Vet J. 2013;91(3):77-82.

47. Roberts E, Boden LA, Ramsey IK. Factors that affect stabilisation times of canine spontaneous hypoadrenocorticism. Vet Rec. 2016;179(4):98.
Veterinary Medicine: Research and Reports

\section{Publish your work in this journal}

Veterinary Medicine: Research and Reports is an international, peer-reviewed, open access journal publishing original research, case reports, editorials, reviews and commentaries on all areas of veterinary medicine. The manuscript management system is completely online and includes a very quick and fair peer-review system.

\section{Dovepress}

Visit http://www.dovepress.com/testimonials.php to read real quotes from published authors. 